\title{
AUTOMATIC CONTINUITY OF MEASURABLE GROUP HOMOMORPHISMS
}

\author{
JONATHAN W. LEWIN ${ }^{1}$
}

\begin{abstract}
It is well known that a measurable homomorphism from a locally compact group $G$ to a topological group $Y$ must be continuous if $Y$ is either separable or $\sigma$-compact. In this work it is shown that the above requirement on $Y$ can be somewhat relaxed and it is shown inter alia that a measurable homomorphism from a locally compact group to a locally compact abelian group will always be continuous. In addition, it is shown that if $H$ is a nonopen subgroup of a locally compact group, then under a variety of circumstances, some union of cosets of $H$ must fail to be measurable.
\end{abstract}

If $f$ is a function from a locally compact group $G$ to a topological space $Y$, and if $m_{G}$ is the left Haar measure of $G$, then we say that $f$ is measurable if $f^{-1}(U)$ is an $m_{G}$-measurable subset of $G$ whenever $U$ is open in $Y$. Certainly, every continuous function must be measurable. When $Y$ is a topological group and $f$ is a homomorphism, then there is a variety of circumstances under which the measurability of $f$ is sufficient to guarantee its continuity. For example, in [2. Theorem 22.18], it is shown that a measurable homomorphism $f$ will certainly be continuous if the group $Y$ is either separable or $\sigma$-compact. It is our purpose here to show that this condition on $Y$ can sometimes be replaced by the weaker condition that $Y$ have an open subgroup which is either separable or $\sigma$-compact. The latter condition is especially interesting because every locally compact group has an open $\sigma$-compact subgroup.

As examples of results that can be obtained, we state the following:

THEOREM 1. Let $f$ be a measurable homomorphism from a locally compact group $G$ to a locally compact group $Y$ and suppose that at least one of the groups $G$ and $Y$ is abelian. Then $f$ is continuous. ${ }^{2}$

THEOREM 2. Let $f$ be a measurable homomorphism from a locally compact group $G$ to a topological group $Y$. Suppose $Y$ has an open normal subgroup $Z$ which is either separable or o-compact and suppose that at least one of the groups $G$ and $Y / Z$ is solvable. Then $f$ is continuous.

Received by the editors April 2, 1982

1980 Mathematics Subject Classification. Primary 43A05, 43A15

Key words and phrases. Locally compact group, abstract harmonic analysis, Haar measure, nonmeasurable set.

'This work was supported in part by the South African Council for Scientific and Industrial Research (C.S.I.R.)

${ }^{2}$ The referee has kindly pointed out that if both $G$ and $Y$ are assumed abelian. Theorem $I$ is proved as Theorem 3.1 in [1]. 
Theorem 2 can be stated more sharply as follows:

THEOREM 3. Let $f$ be a measurable homomorphism from a locally compact group $G$ to a topological group $Y$ which has an open normal subgrroup $Z$ which is either separable or o-compact. Let $H=f^{-1}(Z)$ and suppose that the group $\bar{H} / H$ is solvable. Then $f$ is continuous (and therefore, $\bar{H}=H$ ).

THEOREM 4. A measurable homomorphism from a locally compact group to a discrete group is continuous if and only if its kernel is closed.

The following lemma suggests the method of proof of the above theorems.

LEMMA 1. Let $f$ be a measurable homomorphism from a locally compact group $G$ to a topological group $Y$ and suppose that $Y$ has an open subgroup $Z$ which is either separable or o-compact. Then a (necessary and) sufficient condition for $f$ to be continuous is that the group $f^{-1}(Z)$ be open in $G$.

Proof. The necessity is trivial. Now let $H=f^{-1}(Z)$ and assume that $H$ is an open subgroup of $G$. Since the left Haar measure of $H$ is the restriction to $H$ of the left Haar measure of $G$, we see that for subsets of $H$. measurability with respect to the Haar measures of $G$ and $H$ is the same. Therefore, the restriction of $f$ to $H$ is measurable from $H$ to $Z$ and is therefore continuous by [2. Theorem 22.18]. So $f$ being continuous at the identity 0 of $G$ must be continuous.

In view of Lemma 1 , the above theorems will follow as soon as we have determined sufficient conditions for the group $H=f^{-1}(Z)$ to be open in $G$. In this case the union of any family of cosets of $H$ must be measurable, being the image under $f^{-1}$ of the union of a family of cosets of $Z$ in $Y$. This prompts us to make the following definition.

Definition. A subgroup $H$ of a locally compact group $G$ is said to be totally measurable if given any family of cosets of $H$, the union of the family is measurable with respect to the left Haar measure of $G$.

We conjecture that only an open subgroup can be totally measurable. The following theorem is a step in this direction and in view of Lemma 1, all the above theorems follow at once from it.

THEOREM 5. Let $H$ be a totally measurable normal subgroup of a locally compact group $G$. Then $\bar{H}$ is open in $G$ and the group $\bar{H} / H$ is equal to its commutator subgroup. A fortiori, if $\bar{H} / H$ is solvable (for example, if $G$ is solvable), then $H=\bar{H}$ and consequently, $H$ is open in $G$.

In order to prove Theorem 5, we shall need a few technical results. In what follows, if $H$ is a normal subgroup of a locally compact group $G, \phi$ will denote the natural homomorphism from $G$ to $G / H$. Where appropriate, $m_{G}, m_{H}$ and $m_{G / H}$ will denote left Haar measures of $G, H$ and $G / H$ respectively. All groups will be written additively.

LEMma 2. Let $m$ be a regular measure on a locally compact Hausdorff space $X$ and let $\mathbf{F}$ be a family of nonnegative lower semicontinuous functions on $X$ such that every two members of $\mathbf{F}$ have a common upper bound in F. For each $x$ in $X$, let $g(x)=$ $\sup \{f(x) \mid f$ in $\mathbf{F}\}$. Then we have $\int_{X} g d m=\sup \left\{\int_{X} f d m \mid f\right.$ in $\left.\mathbf{F}\right\}$. 
Proof. One can prove this directly but this result is essentially [2, Theorem 11.33].

LeMma 3. Let $H$ be a closed normal subgroup of a locally compact group $G$. Let $A$ be a subset of $G$ and suppose that $m_{G}(A)=0$. Define $B=\left\{x\right.$ in $G \mid m_{H}((-x+A) \cap H)$ $>0$. Then $B$ is a union of cosets of $H$ and this family $\phi(B)$ of cosets has $m_{G / H}$ measure zero, i.e. $m_{G / H}(\phi(B))=0$.

Proof. It is clear that $B$ is a union of cosets of $H$. Now following [2, $\S \S 15.21-15.23]$ (and see also [3, §2.7.3]), we adjust the Haar measures such that for every $f$ continuous on $G$ with compact support we have

$$
\int_{G} f(x) d m_{G}(x)=\int_{G / H} \int_{H} f(x+y) d m_{H}(y) d m_{G / H}(\phi(x)) .
$$

Using Lemma 2, one can show easily that $(*)$ holds whenever $f$ is nonnegative and lower semicontinuous. In particular, $(*)$ holds for $f=\chi_{U}$ whenever $U$ is open in $G$ and has finite measure. For each natural $n$, choose an open neighborhood $U_{n}$ of $A$ such that $m_{G}\left(U_{n}\right)<1 / n$. If $E$ is the intersection of the sets $U_{n}$, then the dominated convergence theorem implies that

$$
\begin{aligned}
0 & =m_{G}(E)=\int_{G / H} \int_{H} \chi_{E}(x+y) d m_{H}(y) d m_{G / H}(\phi(x)) \\
& =\int_{G / H} m_{H}((-x+E) \cap H) d m_{G / H}(\phi(x))
\end{aligned}
$$

and from this equality, the lemma follows easily.

LEMMA 4. Let $H$ be a closed normal subgroup of a locally compact group $G$. Let $A$ be the union of a family of cosets of $H$ and suppose that $m_{G}(A)=0$. Then $m_{G / H}(\phi(A))=0$.

Proof. This follows at once from Lemma 3 since for every $x$ in $A, H \subseteq-x+A$ and so $m_{H}((-x+A) \cap H) \geqslant m_{H}(H)>0$.

LEMMA 5. Let $H$ be a closed normal subgroup of a locally compact $\sigma$-compact ${ }^{3}$ group $G$. Let $A$ be an $m_{G}$-measurable subset of $G$ and suppose that $A$ is the union of a family of cosets of $H$. Then $\phi(A)$ is $m_{G / H^{-m e a s u r a b l e . ~}}$

Proof. Since $m_{G}$ is $\sigma$-finite on $A$, we can write $A$ in the form $\cup_{n=0}^{\infty} A_{n}$ where $m_{G}\left(A_{0}\right)=0$ and $A_{n}$ is compact for every $n \geqslant 1$. Define $B_{n}=A_{n}+H$ for $n \geqslant 1$ and $B_{0}=A \backslash \cup_{n=1}^{\infty} B_{n}$. Then for $n \geqslant 1$, since $\phi\left(B_{n}\right)=\phi\left(A_{n}\right)$, we see that $\phi\left(B_{n}\right)$ is a compact subset of $G / H$. Furthermore, since $B_{0} \subseteq A_{0}$, we have $m_{G}\left(B_{0}\right)=0$. But $B_{0}$ is a union of cosets of $H$ and we conclude from Lemma 4 that $m_{G / H}\left(\phi\left(B_{0}\right)\right)=0$. Therefore $\phi(A)$ being the union of the sets $\phi\left(B_{n}\right)$, must be $m_{G / H^{r}}$ measurable.

We are now ready to prove the special case of Theorem 5 that occurs when $H$ is closed.

LEMMA 6. Let $H$ be a totally measurable closed normal subgroup of a locally compact group $G$. Then $H$ is open.

\footnotetext{
${ }^{3}$ It is not necessary to assume that $G$ is $\sigma$-compact. However, the proof in the general case is a little more technical and the lemma as stated here is sufficient for our purposes.
} 
Proof. We assume that $H$ is a closed normal subgroup of $G$ but that $H$ is not open. To prove the lemma, we shall show that some union of cosets of $H$ must fail to be $m_{G}$-measurable. Choose an open $\sigma$-compact subgroup $G_{1}$ of $G$ and define $H_{1}=H \cap G_{1}$. Since the interior (in $G$ ) of $H$ is empty, we see that $H_{1}$ is not an open subgroup of the locally compact group $G_{1}$. Therefore the group $G_{1} / H_{1}$ is not discrete and it follows from $[2, \S 16.13]$ that $G_{1} / H_{1}$ has a subset $E$ which is not $m_{G_{1} / H_{1}}$-measurable. $E$ is a family of cosets of $H_{1}$ in $G_{1}$ and the union $A$ of these cosets is not $m_{G_{1}}$-measurable by Lemma 5. Since $G_{1}$ is open in $G$, it follows that $A$ is not $m_{G}$-measurable. But every coset of $H_{1}$ in $G_{1}$ is the intersection with $G_{1}$ of a coset of $H$ in $G$. Therefore, $A$ is of the form $A=B \cap G_{1}$ where $B$ is a union of cosets of $H$ in $G$; and clearly, the set $B$ cannot be $m_{G}$-measurable.

To complete the proof of Theorem 5, we must now concern ourselves with nonclosed subgroups.

LEMMA 7. Let $H$ be a totally measurable dense normal subgroup of a locally compact group $G$. Then the group $G / H$ is equal to its commutator subgroup. A fortiori, if $G / H$ is solvable, we have $H=G$.

Proof. We may write the commutator subgroup of $G / H$ in the form $K / H$ where $K$ is a subgroup of $G$ and to obtain a contradiction we assume that $K$ is a proper subgroup. The group $G / K$ is abelian. If $K$ has countable index in $G$, define $L=K$. Otherwise, as in $[2, \S 16.13]$, choose a subgroup $L / K$ of $G / K$ which has countably infinite index in $G / K$. In either event, $L$ is a proper subgroup of $G$ and has countable index in $G$ and $L$ includes $H$. Being of countable index in $G, L$ cannot be $m_{G}$-locally null. Also, $L$ is the union of a family of cosets of $H$ and therefore, $L$ is $m_{G}$-measurable and it follows that $L$ is open and therefore closed. Therefore, since $H$ is dense in $G$, we have $L=G$ in contradiction to our choice of $L$ as a proper subgroup.

Proof of Theorem 5. Since $H$ is normal in $G$, so is $\bar{H}$. Furthermore, since every coset of $\bar{H}$ is the union of a family of cosets of $H$, we see that $\bar{H}$ is totally measurable and we conclude from Lemma 6 that $\bar{H}$ is an open subgroup of $G$. Therefore, for subsets of $\bar{H}, m_{G}$-measurability and $m_{\bar{H}^{-}}$-measurability are the same and we conclude that $H$ is totally measurable as a subgroup of the locally compact group $\bar{H}$. The result now follows from an application of Lemma 7.

Some results about nonnormal subgroups. The question as to whether a nonnormal totally measurable subgroup must be open, seems very hard to answer. If $H$ is a nonnormal subgroup of $G$, we can still speak of the set $G / H$ of left cosets of $H$ and the natural map $\phi$ from $G$ to $G / H$. With the quotient topology, $G / H$ is locally compact, and is Hausdorff iff $H$ is closed, and discrete iff $H$ is open. Since an analogue of Lemma 7 for nonnormal subgroups seems hopeless, we shall assume that $H$ is closed. Although it is meaningless to speak of a Haar measure on $G / H$, there may still be a measure $m_{G / H}$ on $G / H$ that has much of the behaviour of a left Haar measure and for which the equality (*) in Lemma 3 holds. By [2, Theorem 15.24], the condition for the existence of this measure $m_{G / H}$ is that the modular functions of $G$ and $H$ should agree at every point of $H$. This would hold, for 
example, if $H$ were normal in $G$, and also if $G$ and $H$ were unimodular. We recall that every compact group is unimodular.

With this measure on $G / H$, we can repeat the proofs of Lemmas 3,4 and 5; the chief difference being that we must now refer to left cosets of $H$. Unfortunately, Lemma 6 is more troublesome as it is not obvious that there is a subset $E$ of $G / H$ which is not $m_{G / H^{-}}$-measurable. The following result, which depends on the continuum hypothesis, is the best we can do.

THEOREM 6. Let $H$ be a totally measurable closed subgroup of a $\sigma$-compact locally compact group $G$ of cardinality $|G|=\aleph_{1}=2^{\aleph_{0}}$ and suppose that the modular functions of $G$ and $H$ agree at every point of $H$. Then $H$ is an open subgroup of $G$.

Proof. It is a well-known consequence of the continuum hypothesis that there is no nontrivial finite continuous measure on the $\sigma$-algebra of all subsets of a set of cardinality $\boldsymbol{N}_{1}$. Furthermore, $m_{G / H}$ being nontrivial, must be positive (and finite) on some compact set. To obtain a contradiction, assume that $H$ is not open in $G$. Then it is easy to see that $m_{G / H}$ is continuous and consequently, $G / H$ must have a subset $E$ which is not $m_{G / H}$-measurable. Therefore, by an analogue of Lemma $5, E+H$ is a non- $m_{G}$-measurable union of left cosets of $H$ and we have a contradiction.

For compact groups, more can be proved. Once again, we require the continuum hypothesis.

THEOREM 7. Let $H$ be a totally measurable subgroup of a compact group $G$ of cardinality $|G|=\aleph_{1}=2^{\aleph_{0}}$. Then $H$ is open.

Proof. To obtain a contradiction, we assume that $H$ is not open. Then $m_{G}(H)=0$. Given any subset $E$ of $G / H$ define $\mu(E)=m_{G}\left(\phi^{-1}(E)\right)$. Clearly, $\mu$ is a finite. continuous, nontrivial measure on the $\sigma$-algebra of all subsets of the set $G / H$ and we have reached a contradiction.

Finally, we conclude

THEOREM 8. Let $G$ be a compact group of cardinality $|G|=\boldsymbol{N}_{1}=2^{\boldsymbol{N}_{0}}$ and let $f$ be a measurable homomorphism from $G$ to a topological group $Y$ which has an open subgroup which is either separable or o-compact. Then $f$ is continuous.

\section{REFERENCES}

1. Michael (j. Cowling. Spaces $A_{p}^{4}$ and $L^{p}-L^{4}$ Fourier multipliers, Ph.D. Thesis, Flinders University of South Australia, December 1973.

2. E. Hewitt and K. A. Ross, Abstract harmonics analisis. I, Die Grundlehren der math. Wissenschaften. Springer-Verlag. Berlin, Heidelberg and New York, 1963.

3. W. Rudin. Fourier analysis on groups, Interscience. New York, 1962.

Department of Mathematics, University of Wisconsin, OShKosh, Wisconsin 54901 